\title{
Biologic lung volume reduction therapy for advanced homogeneous emphysema
}

\author{
Y. Refaely*, M. Dransfield ${ }^{\#}$, M.R. Kramer*, M. Gotfried ${ }^{\Uparrow}$, W. Leeds ${ }^{+}$, G. McLennan ${ }^{\S}$, \\ S. Tewari ${ }^{\ddagger}$, M. Krasna** and G.J. Criner ${ }^{\# \#}$
}

ABSTRACT: This report summarises phase 2 trial results of biologic lung volume reduction (BioLVR) for treatment of advanced homogeneous emphysema.

BioLVR therapy was administered bronchoscopically to 25 patients with homogeneous emphysema in an open-labelled study. Eight patients received low dose (LD) treatment with $10 \mathrm{~mL}$ per site at eight subsegments; 17 received high dose (HD) treatment with $20 \mathrm{~mL}$ per site at eight subsegments. Safety was assessed in terms of medical complications during 6-month follow-up. Efficacy was assessed in terms of change from baseline in gas trapping, spirometry, diffusing capacity, exercise capacity, dyspnoea and health-related quality of life.

There were no deaths or serious medical complications during the study. A statistically significant reduction in gas trapping was observed at 3-month follow-up among HD patients, but not LD patients. At 6 months, changes from baseline in forced expiratory volume in $1 \mathrm{~s}$ $(-8.0 \pm 13.93 \%$ versus $+13.8 \pm 20.26 \%)$, forced vital capacity $(-3.9 \pm 9.41 \%$ versus $+9.0 \pm 13.01 \%)$, residual volume/total lung capacity ratio $(-1.4 \pm 13.82 \%$ versus $-5.4 \pm 12.14 \%)$, dyspnoea scores $(-0.4 \pm 1.27$ versus $-0.8 \pm 0.73$ units) and St George's Respiratory Questionnaire total domain scores (-4.9 $\pm 8.3 \mathrm{U}$ versus $-12.2 \pm 12.38$ units) were better with HD than with LD therapy.

BioLVR therapy with $20 \mathrm{~mL}$ per site at eight subsegmental sites may be a safe and effective therapy in patients with advanced homogeneous emphysema.

KEYWORDS: Bronchoscopic lung volume reduction, emphysema therapy, homogeneous emphysema, interventional pulmonology, lung volume reduction surgery

urgical lung volume reduction (LVRS) has been performed for patients with advanced upper lobe predominant (ULP) and homogeneous emphysema, and both groups have experienced clinical benefit [1-3]. However, larger, more consistent improvements in physiological and functional outcomes, and fewer complications, have been reported in patients with ULP disease [4, 5]. In the National Emphysema Treatment Trial (NETT), only patients with ULP disease experienced a survival benefit following LVRS [4, 6, 7]. Serious post-surgical complications, including cardiac ischaemia, arrhythmia and pulmonary embolus, were observed more frequently in patients with homogeneous emphysema [8]. Furthermore, homogeneous patients with an forced expiratory volume in $1 \mathrm{~s}$ (FEV1) $<20 \%$ of predicted were at increased risk of procedural mortality [4].

Recently, the clinical utility of bronchoscopic methods for achieving lung volume reduction has been evaluated in patents with advanced emphysema because these procedures are uniformly safer than surgical volume reduction $[5,9]$. Biologic lung volume reduction (BioLVR) is a novel endobronchial approach that uses a fibrinbased hydrogel to collapse and remodel damaged areas of lung through localised scarring and contraction. The remodelling process is intended to produce therapeutic lung volume reduction more safely than LVRS $[10,11]$. BioLVR has been associated with improvements in physiological, functional and quality of life outcomes in patients with advanced heterogeneous ULP emphysema [9]. This article summarises the results of two small dose ranging phase 2 studies performed to assess the potential safety and efficacy of BioLVR therapy in severe homogeneous emphysema.

\section{METHODS}

\section{Enrolment criteria}

All study participants had severe airflow obstruction, homogeneous emphysema and respiratory

\section{AFFILIATIONS}

${ }^{*}$ Rabin Medical Center, Petah Tiqwa, Israel,

\#University of Alabama Birmingham, Birmingham, AL,

'Pulmonary Associates, Phoenix, AZ, ${ }^{+}$Veritas Clinical Specialties,

Topeka, KS,

${ }^{\S}$ University of lowa, lowa City, IA, ${ }^{f}$ Akron Medical Center, Akron, $\mathrm{OH}$, ${ }^{\star *}$ St. Joseph's Medical Center, Baltimore, MD, and

\#\#Temple University, Philadelphia, PA, USA.

CORRESPONDENCE

Y. Refaely

Division of Cardiothoracic Surgery

Rabin Medical Center

Tel Aviv

Israel

E-mail: Yaelre2@clalit.org.il

Received:

July 072009

Accepted after revision:

Nov 032009

First published online:

Nov 192009 
symptoms despite medical therapy, and were either not eligible for, or had refused, surgical lung volume reduction and lung transplantation. Specific study inclusion/exclusion criteria included: 1) homogeneous emphysema determined by high resolution computed tomography (CT) imaging; 2) persistent symptoms (i.e. a baseline Medical Research Council Dyspnoea (MRCD) score of $\geqslant 2$ ) despite medical therapy; 3 ) age $>40 \mathrm{yrs} ; 4)$ severe airflow limitation, defined as a ratio of FEV1 to forced vital capacity (FVC) $<70 \%$ and an FEV1 $<45 \%$ pred; 5) hyperinflation (total lung capacity (TLC) $>110 \%$ pred and residual volume (RV) $>150 \%$ pred); 6) absence of a diagnosis of alpha-1 anti-protease deficiency; 7) absence of clinically significant pulmonary hypertension, defined as a pulmonary systolic pressure $>45 \mathrm{mmHg}$ (assessed by cardiac echocardiography alone or with right heart catheterisation); 8) abstinence from smoking for $\geqslant 4$ months prior to enrolment; and 9) perfusion of (upper one-third right lung plus perfusions to upper one-third of left lung) $<25 \%$ of total lung perfusion by quantitative scintigraphy scanning [4, 12]. Patients determined to be at high risk of mortality for LVRS were also excluded from participation in this trial [12].

\section{Study design}

Two phase 2 studies were conducted to define the BioLVR dose required to safely and consistently achieve therapeutic lung volume reduction in patients with advanced homogeneous disease. The studies were identical in design except for dosing strategy, and were performed at eight hospitals, seven in the USA and one in Israel. The studies were open-label, nonrandomised and noncontrolled. The first study evaluated bilateral BioLVR treatment at eight treatment sites with $10 \mathrm{~mL}$ per site (enrolment goal 15-20 patients) in patients with advanced homogeneous disease (low dose (LD)). Eight patients enrolled in this study. The second study evaluated bilateral BioLVR treatment at eight treatment sites with $20 \mathrm{~mL}$ per site (enrolment goal 15-20 patients) in patients with advanced homogeneous emphysema (high dose (HD)). 17 patients enrolled in this study. Both protocols were reviewed and approved by the appropriate national regulatory agencies and local ethics committees. All study participants reviewed and signed informed consent forms before enrolment.

Screening evaluations were completed over the course of three separate visits within a 2-week window. Pulmonary function testing was performed during each screening visit, and representative baseline values for each pulmonary function test were determined as the average of three measurements. Exercise capacity, assessed using 6-min walk test (6MWT) distance, was measured on two separate occasions during screening, and baseline defined as the average of these two measurements. Spirometry, plethysmography and 6MWT were performed according to published guidelines [13-16]. Echocardiography, ECG, clinical pathology (haematology, coagulation studies and serum chemistry measurements), radionucleotide lung perfusion scanning and chest $\mathrm{CT}$ imaging were each performed once during screening. CT images were acquired using a standardised acquisition/reconstruction algorithm (spiral acquisition using a multidetector CT scanner with $1 \mathrm{~mm}$ collimation, pitch of 1 , and $0.5 \mathrm{~mm}$ overlap) similar to that used in the NETT $[17,18]$.
BioLVR treatments were administered either in the operating room under general anaesthesia $(10 \mathrm{~mL}$, seven patients; $20 \mathrm{~mL}$, two patients), in the operating room under conscious sedation (10 mL, one patient; $20 \mathrm{~mL}$, one patient), or in the bronchoscopy suite under conscious sedation ( $20 \mathrm{~mL}, 14$ patients), per investigator preference. Conscious sedation regimens included bolus fentanyl and midazolam in 10 patients, low dose propofol infusion in one patient, and intravenous remifentanil infusion in three patients. All procedures were performed using a flexible bronchoscope. Patients were admitted to the hospital for an observation period of up to $48 \mathrm{~h}$ following treatment.

\section{Outcome measures}

The primary end-point of the studies was RV/TLC ratio measured at 3 months following treatment. Treatment success was defined as a statistically significant group mean reduction in RV/TLC from baseline. Treatment efficacy was further assessed in terms of the change from baseline at 3 and 6 months in post-bronchodilator FEV1 and FVC, diffusing capacity of the lung for carbon monoxide (DL,CO), 6MWT distance, MRCD score and St George's Respiratory Questionnaire (SGRQ) health-related quality of life (HRQoL) total domain score, and reduction in RV/TLC at 6 months.

Safety was assessed in terms of the incidence of serious medical complications following BioLVR therapy, and the incidence of post-treatment chronic obstructive pulmonary disease (COPD) exacerbations. A "serious medical complication" was defined as any of the following: 1) death; 2) respiratory failure of $>24 \mathrm{~h}$ duration; 3) pneumothorax; 4) pneumonia; 5) empyema; 6) lung abscess; 7) pulmonary embolus; 8) heart failure; 9) cardiac ischaemia or myocardial infarction; 10) cardiac arrhythmia requiring medical treatment; 11) severe COPD exacerbation requiring admission to an intensive care unit; and 12) a decline in lung physiology posttreatment resulting in permanent loss of function. This list includes significant pulmonary and cardiovascular morbidity prospectively defined by investigators in the NETT, as well as potential procedure-specific adverse complications prospectively identified by BioLVR investigators $[8,9]$.

\section{The BioLVR procedure}

Homogeneous disease was initially assessed by investigators in conjunction with their consulting radiologists. CT DICOM (Digital Imaging and Communications in Medicine) images were then forwarded to the sponsor's medical team for computer analysis and confirmation of homogeneous phenotype by demonstrating that the ratio of the percentage of upper to lower lobe voxels $<-950 \mathrm{HU}$ was between 0.98 and 1.02 (i.e. $1.0 \pm 0.02$ ) using commercially available software (Pulmonary Workstation Plus Software, VIDA Diagnostics, Iowa City, IA, USA) [17]. Any patient not meeting this objective computerbased criterion was considered non-homogeneous, and was excluded from this study. All patients received treatment in the upper lobes or superior segments of the lower lobes. The most damaged areas of lung, identified as those with the lowest HU density and least amount of perfusion, were selected for dosing. In instances where perfusion scan data and CT data were not entirely consistent, CT image results received greater consideration in selecting sites for treatment. 


\begin{tabular}{|c|c|c|c|}
\hline Parameter & LD group & HD group & p-value \\
\hline Subjects $n$ & 8 & 17 & \\
\hline Age yrs & $63.1 \pm 5.06$ & $66.0 \pm 6.75$ & $0.30^{\#}$ \\
\hline Males & $5(62.5)$ & $11(64.7)$ & $>0.99^{\circ}$ \\
\hline$B M I \mathbf{k g} \cdot \mathrm{m}^{-2}$ & $25.6 \pm 6.24$ & $26.6 \pm 5.08$ & $0.69^{\#}$ \\
\hline Smoking history pack-yrs & $58.5 \pm 32.67$ & $63.9 \pm 28.93$ & $0.68^{\#}$ \\
\hline \multicolumn{4}{|l|}{ Oxygen use $\mathrm{L}$} \\
\hline At rest & $0.4 \pm 1.06$ & $1.4 \pm 1.05$ & $0.034^{\#}$ \\
\hline With activity & $0.4 \pm 1.06$ & $2.8 \pm 1.43$ & $<0.001^{\#}$ \\
\hline During sleep & $0.2 \pm 0.71$ & $1.9 \pm 0.95$ & $<0.001^{\#}$ \\
\hline Using any oxygen & $1(12.5)$ & $15(93.8)$ & $<0.001^{\circ}$ \\
\hline $\begin{array}{l}\text { Pulmonary rehabilitation in prior } \\
6 \text { months }\end{array}$ & $2(25.0)$ & $14(82.3)$ & $0.022^{*}$ \\
\hline \multicolumn{4}{|l|}{ Medication use $\%$} \\
\hline Short-acting $\beta$-agonist & 87.5 & 93.7 & NS \\
\hline Short anticholinergic & 25 & 31.3 & NS \\
\hline Long-acting $\beta$-agonist & 100 & 81.3 & NS \\
\hline Long-acting anticholinergic & 75 & 62.5 & NS \\
\hline Inhaled corticosteroid & 87.5 & 87.5 & NS \\
\hline Theophylline preparation & 37.5 & 25 & NS \\
\hline Systemic corticosteroid & 37.5 & 12.5 & NS \\
\hline
\end{tabular}

Data are presented as mean \pm SD or $n(\%)$, unless otherwise stated. LD: low dose; HD: high dose; BMl: body mass index; Ns: nonsignificant. ${ }^{\#}$ : unpaired t-test; ${ }^{\circ}$ : Fisher's exact test.

BioLVR treatments were delivered at the subsegmental airway level, as previously reported [9]. The bronchoscope was advanced into the subsegmental orifice of the treatment site and wedged in position to prevent backflow of reagents. A dual lumen catheter was advanced into the airways with the tip positioned $3-4 \mathrm{~cm}$ beyond the end of the bronchoscope. BioLVR fibrinogen and thrombin solutions were then prepared and delivered through the catheter over 10-15 s. Following administration, the catheter was removed. The bronchoscope was kept in wedge position for $30 \mathrm{~s}$ following instillation of the reagents and then repositioned at the next treatment site.

\section{Data presentation and statistical analysis}

Safety and efficacy results are available for all patients in both treatment groups. Efficacy outcomes at 6 weeks, and 3 and 6 months after treatment were compared with baseline values and reported as change from baseline. Percentage change from baseline is reported for pulmonary function measures, including FEV1, FVC, DL,CO and RV/TLC ratio. Absolute change from baseline is reported for MRCD, 6MWT distance and SGRQ. Statistical significance of the post-treatment change in the primary end-point (RV/TLC ratio at 12 weeks) was assessed by nonparametric testing (Mann-Whitney test). A significant change was defined as $\mathrm{p}<0.05$. Comparisons for all the secondary end-points were performed by nonparametric testing (Kruskal-Wallis one-way ANOVA comparing results at 6, 12 and 24 weeks), and statistical significance was based upon pvalues subject to correction for multiple comparisons using the method of Bonferroni. Correlations between continuous variables were performed using the method of Pearson. Safety outcome measures are summarised using descriptive statistics.

\section{RESULTS}

\section{Patient enrolment and demographic information}

Demographics of patients in the LD and HD treatment groups at the time of enrolment are summarised in table 1 . The two groups were similar with respect to age, sex distribution, smoking history and medication usage. Oxygen use at rest, with activity and during sleep, and the fraction of patients using any supplemental oxygen, was significantly higher in HD compared with LD patients. Furthermore, a significantly larger fraction of HD patients than LD patients had participated in pulmonary rehabilitation within 6 months of study enrolment.

Baseline physiological and functional parameters for the two groups are summarised in table 2 . There were no significant differences in baseline characteristics between the groups, although HD patients had a lower mean 6MWT distance than LD patients prior to therapy $(293 \pm 68.1$ versus $355 \pm 108.7 \mathrm{~m}$; $\mathrm{p}=0.10)$.

The HD arm of the study was fully enrolled. Enrolment in the LD arm of the study was terminated after only eight patients because of lack of response to therapy.

\section{Safety results}

All patients in LD and HD groups tolerated full dose therapy at eight subsegmental sites. There were no procedural complications. Specifically, there were no episodes of bleeding, spillage of hydrogel material, pneumothorax or respiratory failure, or instances in which conscious sedation had to be converted to general anaesthesia because of clinical instability.

Analysis of adverse medical events following BioLVR treatment demonstrated that LD and HD dosing were equally safe. There were no deaths or serious medical complications reported in either treatment group. However, treatment in both groups was associated with significant side-effects. Consistent with its mechanism of action, BioLVR therapy caused a transient inflammatory reaction characterised by

\section{TABLE 2 Baseline physiology}

\begin{tabular}{lccc} 
Parameter & LD group & HD group & p-value \\
\hline Subjects n & 8 & 17 & \\
Post-bronchodilator FEV 1 L & $0.80 \pm 0.194$ & $0.79 \pm 0.197$ & $0.89^{\#}$ \\
Post-bronchodilator FEV1 \% pred & $29.3 \pm 6.89$ & $29.6 \pm 8.59$ & $0.94^{\#}$ \\
FEV1/FVC & $0.31 \pm 0.043$ & $0.31 \pm 0.101$ & $0.84^{\bullet}$ \\
RV L & $4.4 \pm 0.70$ & $4.4 \pm 1.17$ & $0.89^{\#}$ \\
RV \% pred & $197.4 \pm 32.48$ & $195.3 \pm 40.94$ & $0.90^{\#}$ \\
TLC L & $6.8 \pm 1.16$ & $7.4 \pm 1.66$ & $0.39^{\#}$ \\
TLC \% pred & $113.4 \pm 5.10$ & $125.9 \pm 14.35$ & $0.009^{\bullet}$ \\
RV/TLC & $0.65 \pm 0.104$ & $0.60 \pm 0.071$ & $0.18^{\#}$ \\
6MWT distance $\mathbf{m}$ & $355.0 \pm 108.71$ & $293.1 \pm 68.09$ & $0.100^{\#}$ \\
MRCD score & $2.9 \pm 0.83$ & $2.7 \pm 0.48$ & $0.49^{\#}$
\end{tabular}

Data are presented as mean $\pm \mathrm{SD}$, unless otherwise stated. LD: low dose; HD high dose; FEV1: forced expiratory volume in $1 \mathrm{~s} ; \%$ pred: \% predicted; FVC: forced vital capacity; RV: residual volume; TLC: total lung capacity; 6MWT: 6min walk test; MRCD: Medical Research Council dyspnoea. ${ }^{*}$ : unpaired t-test based on equal variances; ${ }^{\bullet}$ : unpaired t-test based on unequal variances. 
TABLE 3 Summary of efficacy response to biologic lung volume reduction treatment

\begin{tabular}{|c|c|c|c|c|c|c|}
\hline \multirow[t]{2}{*}{ Outcome } & \multicolumn{2}{|c|}{6 weeks } & \multicolumn{2}{|c|}{3 months } & \multicolumn{2}{|r|}{6 months } \\
\hline & LD & HD & LD & HD & LD & HD \\
\hline$\Delta F E V_{1}$ (post-bronch) $\%$ & $-1.7 \pm 5.89(\mathrm{NS})$ & $+7.3 \pm 14.92$ (NS) & $+5.6 \pm 5.49$ (NS) & $+11.6 \pm 16.36(p=0.007)^{\#}$ & $-8.0 \pm 13.93$ (NS) & $+13.8 \pm 20.26(p=0.007)^{\#}$ \\
\hline$\Delta \mathrm{FVC}$ (post-bronch) \% & $-1.7 \pm 9.77(\mathrm{NS})$ & $+3.8 \pm 11.97$ (NS) & $+2.4 \pm 8.89$ (NS) & $+7.6 \pm 13.00(\mathrm{NS})$ & $-3.9 \pm 9.41(\mathrm{NS})$ & $+9.0 \pm 13.01$ (NS) \\
\hline$\Delta \mathrm{RV} / \mathrm{TLC} \%$ & $-3.9 \pm 8.26$ (NS) & $-5.0 \pm 6.54$ (NS) & $-5.5 \pm 11.23$ (NS) & $-6.9 \pm 9.60(p=0.008)^{\circ}$ & $-1.4 \pm 13.82(\mathrm{NS})$ & $-5.4 \pm 12.14(\mathrm{NS})$ \\
\hline$\triangle M R C D$ score & $-0.5 \pm 0.76$ (NS) & $-0.6 \pm 0.61$ (NS) & $-0.5 \pm 0.76$ (NS) & $-0.9 \pm 0.93(p=0.001)^{\bullet}$ & $-0.4 \pm 1.27$ (NS) & $-0.8 \pm 0.73(p=0.001)^{\#}$ \\
\hline $\begin{array}{l}\Delta \text { SGRQ total } \\
\text { domain score }\end{array}$ & $-9.4 \pm 9.62$ (NS) & $-9.9 \pm 12.06(p=0.0001)^{\#}$ & $-4.9 \pm 8.39$ (NS) & $-13.0 \pm 14.59(p=0.0001)^{\#}$ & $-4.6 \pm 7.92$ (NS) & $-12.2 \pm 12.38(p=0.0001)^{\#}$ \\
\hline
\end{tabular}

Data are presented as mean $\pm S D$ ( $p$-value). $p$-values were determined from a Kruskal-Wallis test comparing outcomes across the three time points for each treatment group separately. LD: low dose; HD: high dose; $\Delta$ : change; FEV1: forced expiratory volume in $1 \mathrm{~s}$; post-bronch: post-bronchodilator; FVC: forced vital capacity; RV: residual volume; TLC: total lung capacity; DL,CO: diffusing capacity of the lung for carbon monoxide; 6MWD: 6-min walk distance; MRCD: Medical Research Council dyspnoea; SGRQ: St George's Respiratory Questionnaire. * : statistical significance for all secondary outcome measures requires a p<0.007 based upon Bonferroni correction for multiple comparisons; ": comparison for RV/TLC at 12 weeks versus baseline, the primary outcome measure, was performed by Mann-Whitney test.

leukocytosis, fever and malaise within 8-24 h of treatment. Seven of eight LD patients, and 16 of $17 \mathrm{HD}$ patients, manifested at least one of these symptoms. In a smaller subset of patients, transient symptoms of pleuritic chest pain, shortness of breath, nausea and headache were reported. In most cases, this reaction was self limited and resolved within 24$48 \mathrm{~h}$. Hospital length of stay was primarily dictated by the recovery time associated with this reaction. Hospital length of stay was $1.75 \pm 0.71$ days (range $1-3$ days) for LD patients and $1.47 \pm 1.00$ days (range $1-5$ days) for HD patients.

Post-treatment COPD exacerbations were observed in two of eight LD patients (incidence 25\%; 0.5 exacerbations per patient per year), one of which occurred within the first 30 days following treatment and was deemed related to treatment. Post-treatment COPD exacerbations were observed in three of 17 HD patients (incidence 18\%; 0.35 exacerbations per patient per year), two of which occurred within the first 30 days following treatment and were deemed related to treatment. All events were of mild or moderate severity, but four required hospitalisation and intensification of medical treatment.

\section{Efficacy results}

Efficacy responses out to 6 months are summarised in table 3 . HD BioLVR therapy produced a statistically significant reduction in the study primary end-point, $\mathrm{RV} / \mathrm{TLC}$ ratio at 3 month follow-up $(-6.9 \pm 9.6 \% ; p=0.008)$. Improvements following HD therapy at 12 and 24 weeks were also observed in FEV1, FVC, MRCD and SGRQ, although only improvements in FEV1, MRCD, and SGRQ were statistically significant. No significant improvements were observed following LD therapy in any outcome measures. Response patterns of the major physiological and patient reported outcomes at 6 months posttreatment are summarised in figure 1.

Table 4 shows the fraction of patients in the HD and LD treatment groups with improvements in physiological, functional and quality of life measures meeting established minimal clinically important difference criteria. HD therapy produced clinically meaningful improvements in FEV1 and FVC ( $>12 \%$ improvements) in $29 \%$ to $47 \%$ of patients. The fraction of patients demonstrating improvements in spirometry remained stable between 3 and 6 months, confirming durability of response. Clinically significant improvements in dyspnoea (i.e. $\geqslant 1$ unit decline in MRCD score) and HRQoL $(>4$ unit reduction in SGRQ total domain score) were observed in two-thirds to three-quarters of patients out to 6 months following HD therapy. The fraction of patients demonstrating clinically significant improvements in $6 \mathrm{MWT}$ (i.e. $\geqslant 50 \mathrm{~m}$ increase in 6MWT distance) following HD therapy was substantially smaller.

Clinically significant responses following LD treatment were observed only in MRCD and SGRQ scores after treatment.

\section{Radiologic responses to BioLVR}

CT imaging was performed at baseline and 6 weeks after treatment. Both HD and LD groups displayed baseline radiography consistent with severe emphysema assessed in terms of overall tissue density and fraction of lung with tissue density <-950 HU (HD: $-901 \pm 34 \mathrm{HU}, 31 \pm 12 \%$ <-950 HU; LD: $-892 \pm 46 \mathrm{HU}, 26 \pm 18 \%<-950 \mathrm{HU})$. Therapy was not associated with radiological evidence of BioLVR responses outside of preselected treatment sites. There was no radiological evidence of treatment-related mediastinal or pleural pathology. Scarring responses were observed at $47 \pm 19 \%$ of LD and at $60 \pm 20 \%$ of HD treatment sites. Among the combined cohort of 25 patients, the number of treatment sites demonstrating radiological evidence of remodelling correlated significantly with percentage improvements in FEV1 at $3(\mathrm{r}=0.49, \mathrm{p}=0.01, \mathrm{n}=25)$ and 6 $(\mathrm{r}=0.56, \mathrm{p}=0.004, \mathrm{n}=25)$ month follow-up.

CT images from selected patients are presented in figures 2-5. These images show the peripheral subsegmental upper lobe atelectasis characteristic of BioLVR responses. On a per site basis, scarring reactions were more extensive following $20 \mathrm{~mL}$ per site treatment (figs 2 and 3) than $10 \mathrm{~mL}$ per site treatment (figs 4 and 5). 

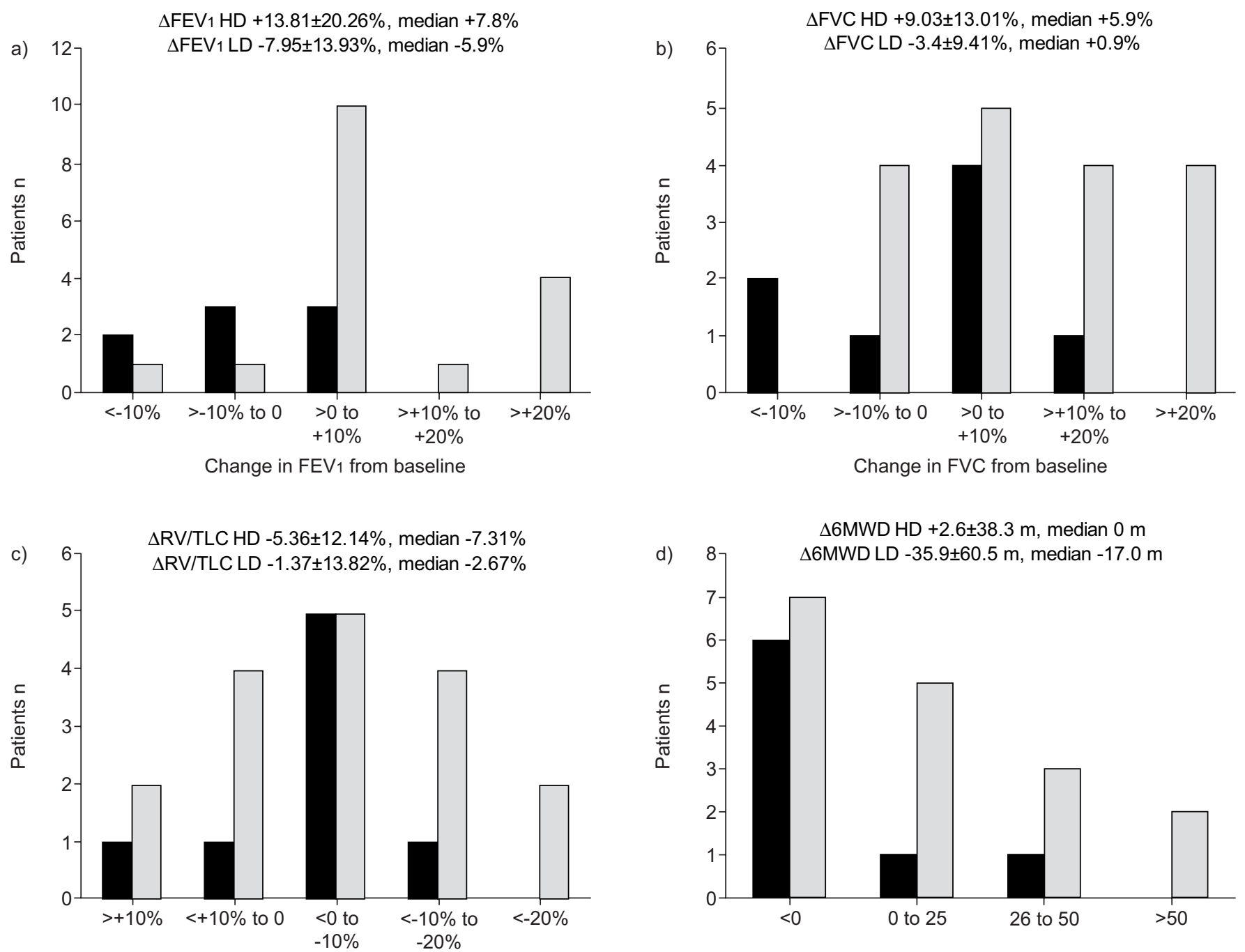

Change in RV/TLC from baseline
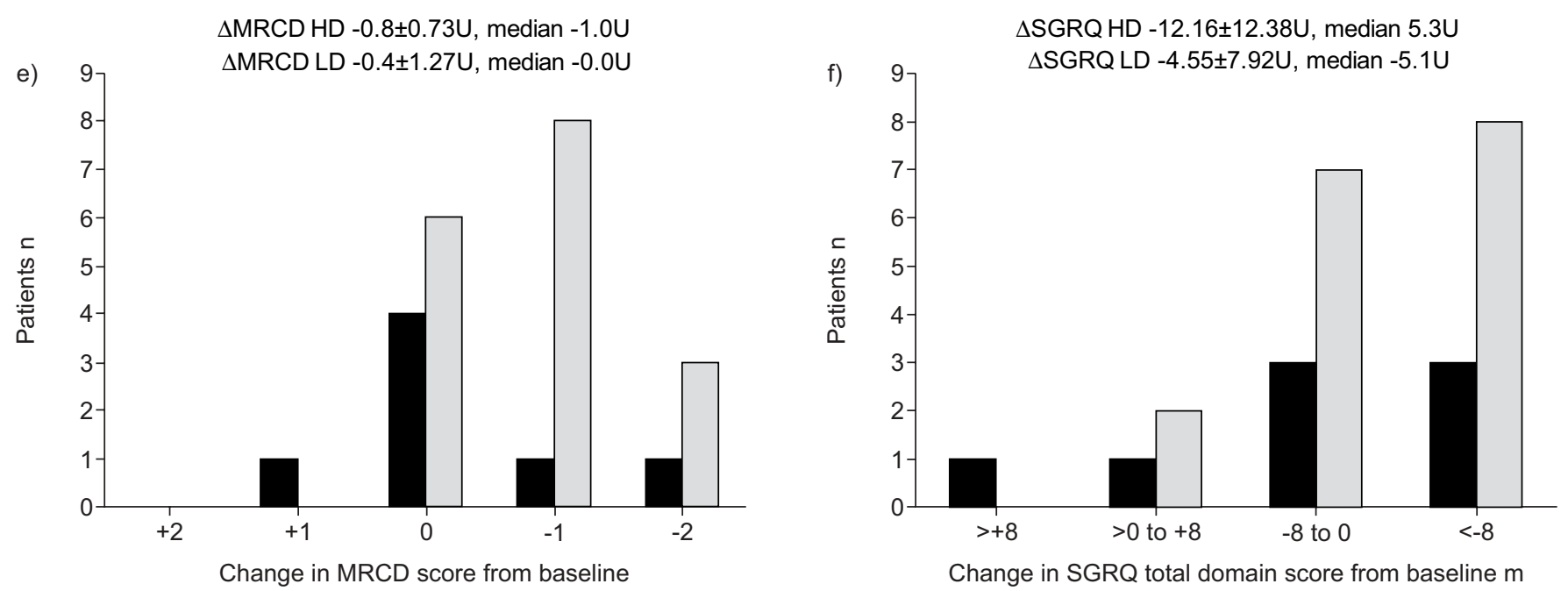

FIGURE 1. Summary of responses to low (LD; $\mathbf{a})$ and high (HD; - ) dose biologic lung volume reduction therapy at 6 months after treatment. Group responses are also summarised as mean \pm SD and median values. FEV1: forced expiratory volume in $1 \mathrm{~s}$; FVC: forced vital capacity; RV: residual volume; TLC: total lung capacity; 6MWD: 6-min walk distance; MRCD: Medical Research Council dyspnoea; SGRQ: St George's Respiratory Questionnaire. 


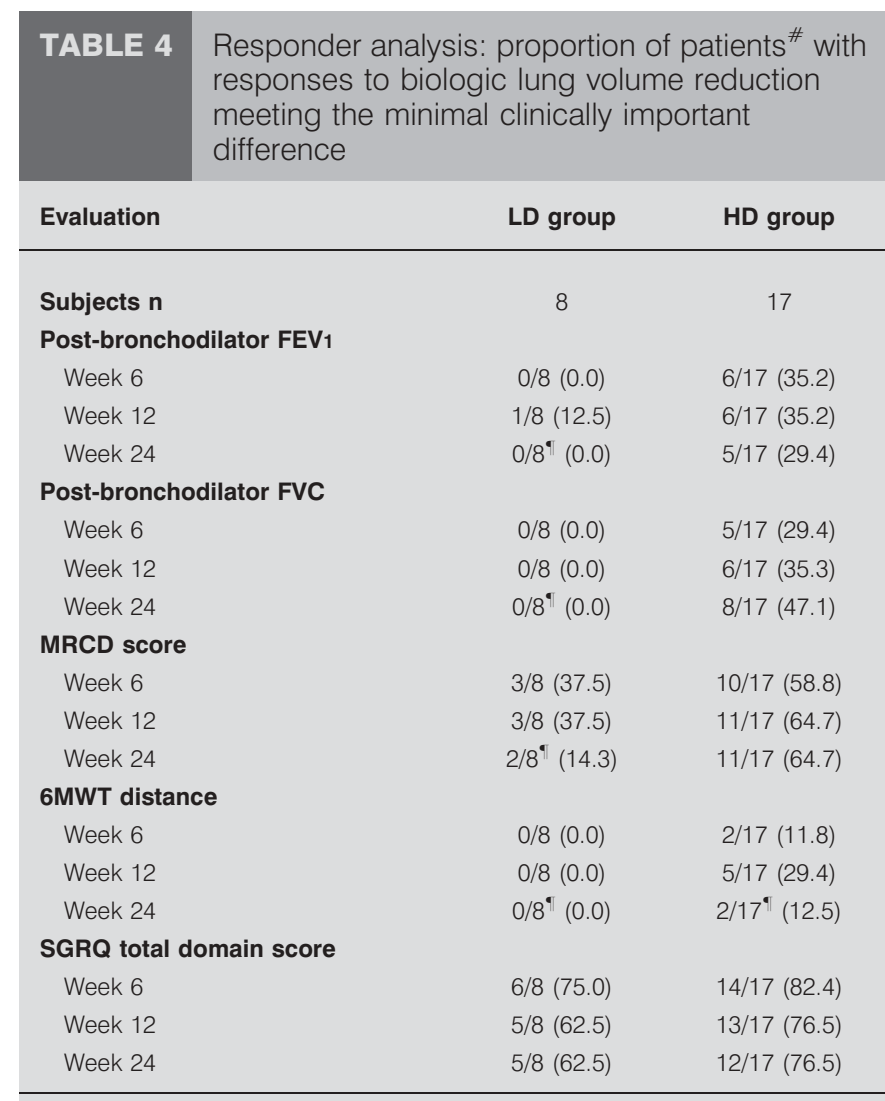

Data are presented as $\mathrm{n} / \mathrm{N}(\%)$, unless otherwise stated. LD: low dose; HD: high dose; FEV1: forced expiratory volume in $1 \mathrm{~s}$; FVC: forced vital capacity; MRCD: Medical Research Council dyspnoea; 6MWT: 6-min walk test; SGRQ: St George's Respiratory Questionnaire. FEV1 and FVC response based on a 12\% improvement (increase) from baseline. Response for functional assessments defined as increase of $50 \mathrm{~m}$ for the 6MWT distance, a decrease of 1 unit for MRCD score and a decrease of 4 units for SGRQ. \#: with homogeneous disease $(n=25) ;{ }^{\bullet}$ : data imputed for one patient from 12 week time point.

\section{DISCUSSION}

BioLVR is a novel bronchoscopic therapy for reducing lung hyperinflation in advanced emphysema. BioLVR treatment involves endobronchial administration of a hydrogel that flows into the alveolar compartment and polymerises, collapsing enlarged airspaces and triggering a localised inflammatory reaction that remodels and contracts diseased emphysematous lung tissue. Prior results have demonstrated the safety and potential efficacy of BioLVR in patients with advanced ULP emphysema $[9,19]$. In a cohort of 22 patients, BioLVR therapy with $20 \mathrm{~mL}$ of hydrogel at eight subsegmental sites was associated with improvements in FEV1, FVC, RV/TLC ratio, dyspnoea scores and HRQoL out to 6 months following treatment. The present study shows that treatment can be performed safely with physiological and functional benefits in patients with advanced homogeneous emphysema, although responses reported here are smaller than those observed in patients with upper lobe heterogeneous disease.

Theoretical considerations suggest that volume reduction therapy could benefit patients with advanced emphysema independent of whether the distribution of disease is homogeneous or heterogeneous [20]. Resection of hyperinflated lung tissue and normalisation of the mechanical relationship between the oversized lung and chest wall increases lung recoil, vital capacity and expiratory flows, and restores the respiratory muscles to a more normal configuration. In practice, LVRS has been associated with reduced efficacy and a higher incidence of complications in homogeneous compared with heterogeneous ULP emphysema and is generally avoided in patients with homogeneous emphysema [8].

The results presented here suggest that BioLVR may represent a safer, potentially effective alternative to LVRS in patients with advanced homogeneous emphysema. Although encouraging, these results must be considered preliminary, and interpreted with caution. Selection bias exists in this study as enrolment was limited specifically to patients not eligible for, or refusing, surgical volume reduction and transplantation. Furthermore, confirmation of these initial findings in a larger, randomised controlled trial with longer follow-up is needed to fully assess the utility of BioLVR therapy in patients with advanced homogeneous emphysema. It is also important to note that patients identified by NETT investigators as being at high risk of death following LVRS (homogeneous disease with FEV1 $<20 \%$ pred) were specifically excluded from this study. Thus, the potential for BioLVR to address this important unmet need was not addressed in this study.

BioLVR treatment was associated with a predictable, acute, self-limited mild-to-moderate inflammatory reaction in all patients. This "flu-like" reaction resolves within 24-48 h, responds to general supportive medical care, and is reasonably well tolerated. Side-effects were similar to those previously reported in patients who received BioLVR at eight subsegmental sites for treatment of ULP emphysema [9].

The safety profile of BioLVR reported here in advanced homogeneous emphysema compares favourably to LVRS and other endobronchial lung volume reduction techniques. LVRS is associated with a $5-20 \% 90$ day mortality and endobronchial valve therapy $(n=98)$ and airway bypass $(n=36)$ with an approximately $1-3 \% 90$ day mortality [8, 21, 22]. By comparison, no deaths were observed among the 25 patients treated with BioLVR in this study. Serious pulmonary and cardiovascular complications within 30 days of treatment have been reported in up to $49 \%$ of LVRS patients and $27 \%$ of airway bypass patients $[9,16]$. In the current study none of the 25 patients treated with BioLVR experienced serious medical complications out to 6 months.

Improvements in physiology after BioLVR therapy follow a pattern consistent with the mechanism proposed by FESSLER et al. [20] to explain physiological responses to surgical volume reduction. Among LD and HD patients combined, percentage change in both FEV1 $(\mathrm{n}=25 ; \mathrm{r}=-0.614, \mathrm{p}<0.001)$ and FVC $(n=25 ; r=-0.744, p<0.001)$ at 6 months correlated with percentage reduction in gas trapping ( $R V / T L C$ ratio), suggesting that the basis for improvement following BioLVR therapy in patients with advanced homogeneous emphysema is lungchest wall resizing. This physiological mechanism of action is further supported by CT imaging, which shows a statistically significant, although limited, correlation between the number 

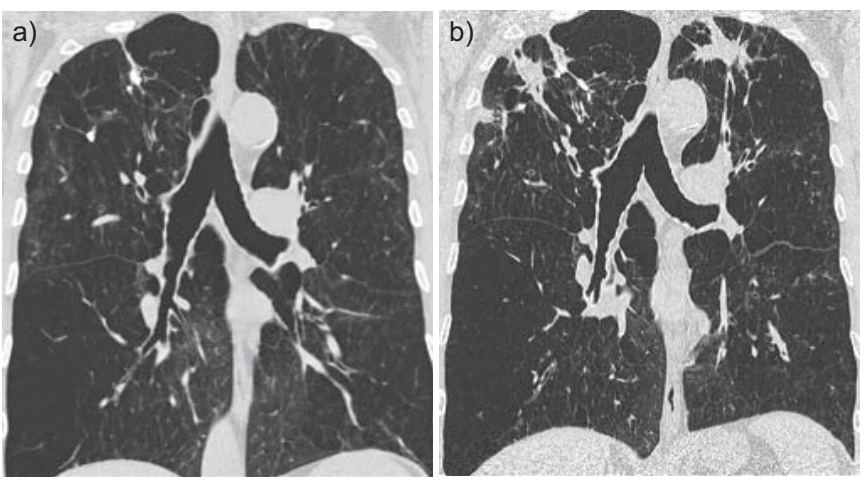

FIGURE 2. a) Baseline and b) week-6 post-biologic lung volume reduction (BioLVR) high dose therapy computed tomography (CT) responses. Coronal multiplanar reformation CT images in a patient treated at eight sites with $20 \mathrm{~mL}$ of BioLVR hydrogel per site, four treatments in each upper lobe. Scarring responses were observed at six of the eight sites. The patient experienced a $+21 \%$ improvement in forced expiratory volume in $1 \mathrm{~s}, \mathrm{a}+9 \%$ improvement in forced vital capacity and a -22 unit improvement in St George's Respiratory Questionnaire total domain score.

of treatment sites that demonstrate atelectasis and the improvements in pulmonary function.

Imaging studies confirm the ability to target BioLVR therapy to specific anatomic locations via the endobronchial route. Neither LD nor HD therapy was associated with radiographic evidence of mediastinal, pleural or parenchymal changes beyond those associated with treatment-site remodelling. Radiographic responses were observed more consistently, and were larger following HD therapy compared with LD therapy (figs 2-5).

Although this phase 2 study was open labelled and uncontrolled, results support the potential efficacy and safety of BioLVR therapy in patients with advanced homogeneous emphysema
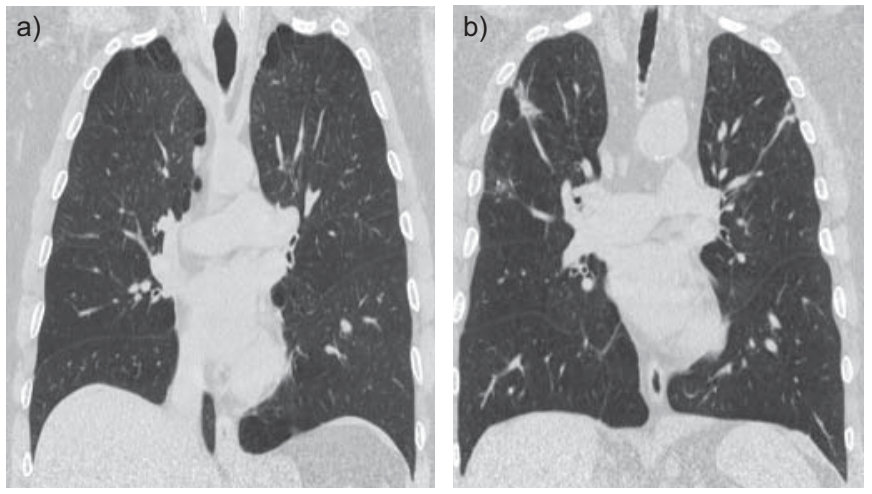

FIGURE 4. a) Baseline and b) week-6 post-biologic lung volume reduction (BioLVR) low dose therapy computed tomography (CT) responses. Coronal multiplanar reformation CT images in a patient treated at eight sites with $10 \mathrm{~mL}$ of BioLVR hydrogel per site, four treatments in each upper lobe. Scarring responses were observed at three of the eight sites. The patient experienced a $+1 \%$ improvement in forced expiratory volume in $1 \mathrm{~s}, \mathrm{a}+6 \%$ improvement in forced vital capacity and a -9 unit improvement in St George's Respiratory Questionnaire total domain score.

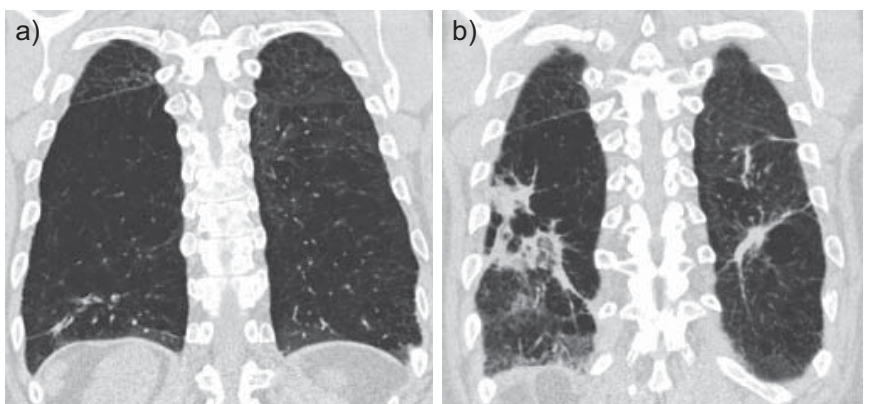

FIGURE 3. a) Baseline and b) week-6 post-biologic lung volume reduction (BioLVR) high dose therapy computed tomography (CT) responses. Coronal multiplanar reformation CT images in a patient treated at eight sites with $20 \mathrm{~mL}$ of BioLVR hydrogel per site, four treatments in each lower lobe superior segment. Scarring responses were observed at six of the eight sites. The patient experienced a $+37 \%$ improvement in forced expiratory volume in $1 \mathrm{~s}, \mathrm{a}+14 \%$ improvement in forced vital capacity and a -19 unit improvement in St George's Respiratory Questionnaire total domain score.

and persistent respiratory symptoms. Improvements at followup time points in objective, largely effort-independent physiological outcomes, including FEV1 and RV/TLC, cannot be explained by a placebo effect. Potential therapeutic effectiveness is further supported by evidence of a dose-response relationship across LD and HD patients in physiological and patient-reported outcomes.

In summary, BioLVR therapy is one of several endobronchial approaches being developed to treat lung hyperinflation in advanced emphysema. In this small study, safety and efficacy responses following HD therapy in patients with homogeneous emphysema compared favourably to LVRS and endobronchial airway bypass, and the responses appeared durable out to 6 months of follow-up. Additional, randomised controlled studies in a larger number of patients are required to confirm the preliminary findings presented here, but initial results suggest that BioLVR therapy may represent a new treatment for patients with advanced homogeneous emphysema.
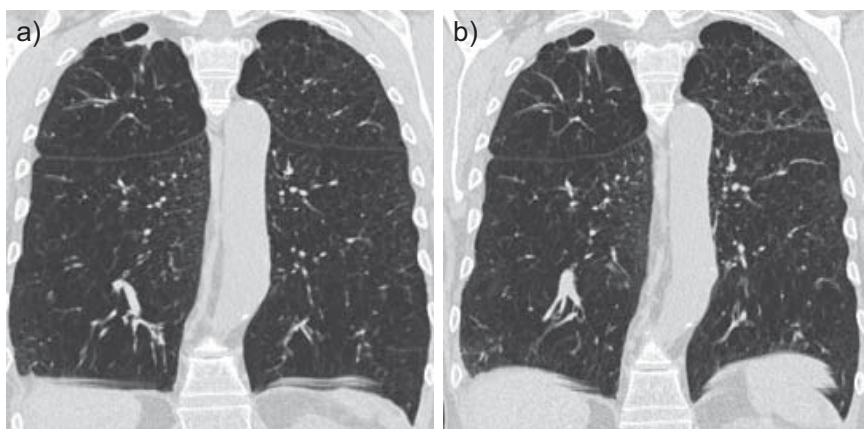

FIGURE 5. a) Baseline and b) week-6 post-biologic lung volume reduction (BioLVR) low dose therapy computed tomography (CT) responses. Coronal multiplanar reformation CT images in a patient treated at eight sites with $10 \mathrm{~mL}$ of BioLVR hydrogel per site, four treatments in each upper lobe. Scarring responses were observed at three of the eight sites. The patient experienced a $-3 \%$ change in forced expiratory volume in $1 \mathrm{~s}, \mathrm{a}+4 \%$ improvement in forced vital capacity and a -15 unit improvement in St George's Respiratory Questionnaire total domain score. 


\section{STATEMENT OF INTEREST}

A statement of interest for this study can be found at www.erj. ersjournals.com $/ \mathrm{misc} /$ statements.dtl

\section{BIBLIOGRAPHY}

1 Date H, Goto K, Souda R, et al. Predictors of improvement in FEV1 (forced expiratory volume in 1s) after lung volume reduction surgery. Surg Today 2000; 30: 328-332.

2 DeCamp MM Jr, Lipson D, Krasna M, et al. The evaluation and preparation of the patient for lung volume reduction surgery. Proc Am Thorac Soc 2008; 5: 427-431.

3 McKenna RJ Jr, Gelb A, Brenner M. Lung volume reduction surgery for chronic obstructive pulmonary disease: where do we stand? World J Surg 2001; 25: 231-237.

4 Fishman A, Martinez F, Naunheim K, et al. A randomized trial comparing lung-volume-reduction surgery with medical therapy for severe emphysema. N Engl J Med 2003; 348: 2059-2073.

5 Wood DE. Results of lung volume reduction surgery for emphysema. Chest Surg Clin N Am 2003; 13: 709-726.

6 Martinez FJ, Chang A. Surgical therapy for chronic obstructive pulmonary disease. Semin Respir Crit Care Med 2005; 26 167-191.

7 Ramsey SD, Berry K, Etzioni R, et al. Cost effectiveness of lungvolume-reduction surgery for patients with severe emphysema. $N$ Engl J Med 2003; 348: 2092-2102.

8 Criner GJ, Sternberg AL. National Emphysema Treatment Trial: the major outcomes of lung volume reduction surgery in severe emphysema. Proc Am Thorac Soc 2008; 5: 393-405.

9 Criner GJ, Pinto-Plata V, Strange C, et al. Biologic lung volume reduction in advanced upper lobe emphysema: phase 2 results. Am J Respir Crit Care Med 2009; 179: 791-798.

10 Ingenito EP, Berger RL, Henderson AC, et al. Bronchoscopic lung volume reduction using tissue engineering principles. Am J Respir Crit Care Med 2003; 167: 771-778.
11 Ingenito EP, Wood DE, Utz JP. Bronchoscopic lung volume reduction in severe emphysema. Proc Am Thorac Soc 2008; 5: 454-460.

12 National Emphysema Treatment Trial Research Group. Patients at high risk of death after lung-volume-reduction surgery. $N$ Engl J Med 2001; 345: 1075-1083.

13 Wanger J, Clausen JL, Coates A, et al. Standardisation of the measurement of lung volumes. Eur Respir J 2005; 26: 511-522.

14 Aggarwal AN, Agarwal R. The new ATS/ERS guidelines for assessing the spirometric severity of restrictive lung disease differ from previous standards. Respirology 2007; 12: 759-762.

15 ATS Committee for Proficiency Standards for Clinical Pulmonary Function Laboratories. ATS statement: guidelines for the six-minute walk test. Am J Respir Crit Care Med 2002; 166: 111-117.

16 Brooks D, Solway S, Gibbons WJ. ATS statement on six-minute walk test. Am J Respir Crit Care Med 2003; 167: 1287.

17 Washko GR, Criner GJ, Mohsenifar Z, et al. Computed tomographicbased quantification of emphysema and correlation to pulmonary function and mechanics. COPD 2008; 5: 177-186.

$18 \mathrm{Kohl} \mathrm{G}$. The evolution and state-of-the-art principles of multislice computed tomography. Proc Am Thorac Soc 2005; 2, 470-476: 499-500.

19 Reilly J, Washko G, Pinto-Plata V, et al. Biological lung volume reduction: a new bronchoscopic therapy for advanced emphysema. Chest 2007; 131: 1108-1113.

20 Fessler HE, Scharf SM, Ingenito EP, et al. Physiologic basis for improved pulmonary function after lung volume reduction. Proc Am Thorac Soc 2008; 5: 416-420.

21 Yim AP, Hwong TM, Lee TW, et al. Early results of endoscopic lung volume reduction for emphysema. J Thorac Cardiovasc Surg 2004; 127: 1564-1573.

22 Cardoso PF, Snell GI, Hopkins P, et al. Clinical application of airway bypass with paclitaxel-eluting stents: early results. J Thorac Cardiovasc Surg 2007; 134: 974-981. 